\title{
¿Qué es relevante históricamente? Pensamiento histórico a través de las narrativas de los estudiantes universitarios ${ }^{1}$
}

\author{
Alejandro Egea Vivancos² \\ Laura Arias Ferrer ${ }^{2}$
}

\section{Resumen}

El objetivo es conocer a qué acontecimientos históricos atribuyen relevancia los estudiantes del Grado de Educación Primaria y en qué términos lo hacen. Para ello se analizan los patrones de pensamiento histórico que poseen los futuros docentes. Se centra la atención en el estudio de la denominada relevancia histórica. A través de pequeñas narrativas se han analizado cuáles son las capacidades del futuro docente en relación a este concepto básico del pensamiento histórico, lo cual aporta interesantes indicios acerca de cómo el alumnado entiende la historia y bajo qué premisas la narra. Para ello se han procesado 107 pequeñas narrativas construidas grupalmente por 520 individuos, todos ellos estudiantes universitarios del grado de Educación Primaria. Los textos se recopilaron durante tres cursos académicos y se analizaron siguiendo una metodología cualitativa a partir de la categorización de sus contenidos. Los resultados demuestran que los futuros docentes cuentan con unos conocimientos históricos mínimos, la mayoría de ellos limitados a los episodios que marcan los cambios de etapa histórica. Estos acontecimientos de transición entre etapas son para ellos los más relevantes de la historia de la humanidad obviando otros. Algunas de las implicaciones para su futura labor como docentes son una gran limitación de los contenidos a enseñar o una excesiva dependencia del libro de texto, entre otras. Esto lleva al desarrollo de enseñanzas basadas en la memorización y en la ausencia de reflexión sobre la propia narración histórica obviando por tanto el análisis de la relevancia de algunos eventos históricos.

\section{Palabras clave}

Historia - Enseñanza de la historia - Educación primaria - Formación del profesorado.

\footnotetext{
1 - Investigación realizada en el marco del Proyecto La evaluación de las competencias y el desarrollo de capacidades cognitivas sobre Historia en Educación Secundaria Obligatoria (EDU2015-65621-C3-2-R), financiado por el Ministerio de Economía y Competitividad de España / Fondos FEDER y el Programa Jiménez de la Espada de Movilidad, Cooperación e Internacionalización de la Fundación Séneca, dentro del plan de Ciencia, Tecnología e Innovación de la Región de Murcia (España).

2 - Universidad de Murcia, Murcia, España.

Contactos: alexegea@um.es; larias@um.es
}

DOl: http://dx.doi.org/10.1590/S1678-4634201709168641 


\section{What is historically significant? Historical thinking through the narratives of college students}

\section{Abstract}

This study aims to analyze the historical relevance given by Basic Education students to historical events and in what ways they do it. For that, standards of historical thinking held by those future teachers are analyzed. Focus is on the so called historical significance. Through short account, we analyzed the abilities of the future teacher in relation to this basic concept of the historical thinking, which brings interesting signs about how students understand history and the underlying assumptions to narrate it. For that purpose, we processed 107 short narratives written by 520 individuals, all of them college students of Basic Education working in groups. The texts were collected during three academic courses and were analyzed with qualitative methodology based on the categorization of their contents. The results show that these future teachers have minimum historical knowledge, most of it limited to events that are the landmarks of changes in a historical period. These events of transition between historical periods are, for them, the most significant in the history of humankind to the detriment of others. Some of the implications for their future work as teachers include a great limitation of the contents to be taught or an excessive dependence on the textbook. This leads to the development of a teaching based on memorization and the lack of reflection of the historical narration itself to the detriment of the analysis of the importance of some historical events.

\section{Keywords}

History - History teaching - Basic education - Teacher training.

\section{Introducción}

Las investigaciones sobre el conocimiento histórico de los estudiantes, especialmente aquellas que se detienen en la forma en la que los alumnos pueden y deben comprender la historia, es una de las líneas de investigación más en boga en el mundo anglosajón (HENRÍQUEZ; PAGĖS, 2004; LEVSTIK, 2011). Dentro de esta línea, en los últimos años se ha incidido en la necesidad de enseñar a los alumnos a pensar históricamente (SEIXAS, 1996; WINEBURG, 2001; SEIXAS; PECK, 2004; WINEBURG, 2007; LÉVESQUE, 2008; VANSLEDRIGHT, 2011; 2014; SEIXAS; MORTON, 2013; GÓMEZ; ORTUÑO; MOLINA, 2014; DOMÍNGUEZ, 2015). Es un mantra que se repite hasta la saciedad en la bibliografía especializada en didáctica de la historia pero que, desgraciadamente, no por mucho repetirlo, se hace más efectivo en la cotidianeidad de las aulas. Posiblemente el salto de la investigación al aula pueda y deba darse a través de la formación del futuro profesorado. ¿Pero poseen los futuros docentes las bases sobre las que construir este pensamiento histórico? ¿Cómo es este 
conocimiento de la historia en relación a algunas de las categorias de metaconceptos del pensamiento histórico? Tras haber realizado una aproximación general sobre qué recuerdan sobre historia los futuros docentes de Primaria (ARIAS; EGEA, 2015), en esta ocasión se pretende focalizar el análisis en una de esas competencias que conforman el pensamiento histórico, la denominada como relevancia histórica, más conocida por su término en inglés como historical significance, por considerar que se trata de una de las categorías clave para conocer cuál es la percepción que los futuros docentes poseen sobre la historia. Al fin y al cabo, la comprensión de ésta se muestra clave para entender el carácter de variabilidad que posee la propia disciplina. Así, un hecho histórico es relevante o significativo dependiendo de cuál sea el objetivo que se persiga, el marco geográfico en el que uno se mueva, etc. Esta tarea pasa porque el alumnado conozca los denominados como contenidos sustantivos de la historia para poder trascender a la comprensión de los metaconceptos. El problema es que estos contenidos más conceptuales se aprenden (y a veces se recitan) sin aplicar sobre ellos una propia reflexión acerca de su presencia o selección dentro de la narrativa de la historia.

Por todo ello, se cree que este análisis diagnóstico será clave para poder marcar un punto de partida para promover estrategias que incentiven un giro en la forma de enseñar y aprender la historia, y que estas nuevas formas han de comenzar por la propia reflexión sobre la idoneidad de la selección de los contenidos a impartir.

\section{La relevancia histórica}

Los denominados como contenidos sustantivos (substantive concepts), de primer orden o contenidos conceptuales son uno de los componentes clave del pensamiento histórico. Se trata de fechas, datos, personajes, conceptos, etc., que son la base sobre la que construir a posteriori las diferentes destrezas estratégicas para dar significado a ese conocimiento, entendidas como elementos estructurales, contenidos de segundo orden o metaconceptos (second-order concepts, organizing concepts), y que se concretan en competencias para responder y significar cuestiones históricas y para entender el pasado tal y como se hace en la investigación histórica (SEIXAS, 1996; CERCADILLO, 2000; WINEBURG, 2001; LÉVESQUE, 2008; VANSLEDRIGHT, 2011; 2014).

Muchos son los autores que han considerado la relevancia histórica como una destreza básica dentro de esos conceptos estructurales (SEIXAS, 1994, 1996; CERCADILLO, 2000; 2006; SEIXAS; PECK, 2004; LÉVESQUE, 2005; 2008; SEIXAS; MORTON, 2013). La historical significance, relevancia histórica o, si se quiere, lo históricamente determinante, consiste en que nuestros alumnos sepan determinar y comprender qué hechos históricos han sido claves en la historia y por qué. De este modo, podrán responder a la eterna pregunta en torno a la utilidad de estudiar el pasado para su formación. ¿Pero qué implica este concepto?

Es obvio que estudiarlo todo es imposible y que hay que escoger, obligatoriamente, entre todas esas piezas del corpus que conforman el pasado qué es lo que verdaderamente merece o no la pena (SEIXAS, 1994). Muchos docentes se encuentran en alguna ocasión en la tesitura de tomar la decisión de escoger qué períodos o acontecimientos se enseñan a los alumnos. Y ante esa situación, se debe reflexionar sobre qué hace a un acontecimiento relevante o no, así como la necesidad de transmitir esta importancia al propio alumnado. Ya Partington (1980 apud PHILLIPS, 2002) estableció una serie de factores al respecto. 
Así, la importancia para la gente contemporánea al hecho, la profundidad a la hora de influir en las vidas de las gentes, la cantidad de personas que se vieron afectadas, la duración de sus consecuencias y, por último, la relevancia, es decir, el grado en el que ese concreto evento contribuye a comprender el presente, son algunos de esos factores. En este mismo sentido, para Lomas (1990) era básico incidir también, por ejemplo, en que algunos acontecimientos eran más importantes que otros, que esta importancia cambiaba con el paso del tiempo, que diferentes personas podían tener distintas ideas sobre qué había sido más determinante en la historia, que acontecimientos aparentemente insignificantes podian ser extremadamente simbólicos o que un evento podía adquirir más significatividad conforme poseyera más conexiones con otros hechos.

Sin embargo, y como se podrá comprobar en los resultados obtenidos, aquello que inicialmente consideran los estudiantes como determinante en la historia está desgraciadamente muy lejos de todas estas consideraciones. Y es que, y siguiendo la reflexión de Seixas (1997) al respecto, a pesar de contar cada año con profesores y libros de texto que seleccionan para el alumnado qué es lo determinante de la historia (siguiendo o no estos criterios), al final se produce un filtrado y una criba en la mente del estudiante entre lo que recuerda, olvida, añade, modifica, etc. Ya ha habido ciertas aproximaciones en otros ámbitos educativos sobre dicha cuestión, procurando determinar qué acontecimientos son más relevantes para los estudiantes. Así, ya se cuenta con información para estudiantes de Educación Secundaria o Bachillerato en el caso de Canadá (SEIXAS, 1994; LÉVESQUE, 2005), Estados Unidos (BARTON; LEVSTIK, 1998) o incluso una comparativa entre estudiantes de Reino Unido y España (CERCADILLO, 2000; 2006). Pero como novedad, en esta ocasión, se presentan los resultados de una investigación sobre el ámbito español y, en concreto, sobre aquellos que van a ser en cuestión de unos pocos años profesores de la Educación Primaria en nuestro país.

\section{Marco metodológico}

\section{Objetivos}

La pregunta de investigación que pretende contestar este artículo es la que le da título: ¿Qué es relevante históricamente para los futuros docentes de Educación Primaria? Dicha pregunta nos permite enmarcar el objetivo planteado centrado en: analizar a qué acontecimientos históricos atribuyen relevancia histórica los futuros docentes de Educación Primaria y en qué términos lo hacen.

Este tipo de análisis se muestra claramente fundamental para poder determinar cómo concibe la Historia hoy el que será el profesorado de Educación Primaria del mañana ya que, sin duda, esta concepción va a determinar la manera en la que estos desarrollen su acción docente ulterior.

\section{Contexto y participantes}

Durante tres cursos académicos (2012-2015) se propuso una actividad a alumnos de $2 .^{\circ}$ curso de Educación Primaria de la Universidad de Murcia (EGEA; ARIAS, 2015). El número total de participantes en la actividad ascendió a unos 520 individuos. Sus edades 
oscilaban entre los 19 y los 35 años, de los que una buena parte de ellos (casi un 75\%) contaba con 19 y 20 años. El muestreo por tanto fue por conveniencia, no probabilístico y los estudiantes escogidos se corresponden con los grupos a los que se les impartía clase.

En pequeños grupos, los estudiantes componían pequeñas estrofas sobre períodos históricos, a modo de rap. El número total de composiciones obtenidas fue de 107. Cabe recordar que para estos alumnos, la asignatura Historia de España era troncal y, por lo tanto, obligatoria en $2 .^{\circ}$ de Bachillerato, por lo que de manera casi general el último contacto del grupo con la historia, académicamente hablando, había sido hacía apenas dos años.

\section{Procedimiento}

Los textos recopilados $(n=107)$ fueron codificados de manera correlativa [T001-T107]. A través de un método de investigación cualitativo se analizaron las estrofas compuestas de manera grupal. Cada grupo construyó libremente sus estrofas a través de los recuerdos colectivos. Tras el correspondiente tratamiento informático de los textos mediante el programa de análisis de datos cualitativos Atlas.ti (v.7), se establecieron una serie de unidades, categorías y subcategorías (STRAUSS; CORBIN, 1998). Las unidades establecidas hacían alusión al modo en el que el alumnado afrontaba la tarea narrativa de la Historia, diferenciándose entre enumeración (mera secuencia de hechos inconexos), descripción (selección de un hecho y descripción del mismo) y relatos (donde el alumnado generaba una trama o se incluía como protagonista en la acción). La segunda unidad de análisis se correspondía con el periodo cronológico al que la composición se adscribía. Así, los diferentes textos fueron clasificados en base a periodo y subperiodo histórico, $\mathrm{y}$, a partir de ahí, según su categoría principal y subcategoría. Dentro de las categorías se distinguieron episodios y acontecimientos, principales protagonistas y temas. Los temas fueron divididos a su vez en política, sociedad, economía, religión o cultura y arte. Para este análisis concreto se hace especial hincapié en las categorías de eventos y protagonistas, cuyas características aportan los indicios clave para entender el grado de relevancia que el alumnado otorga a lo narrado. Sobre el resto de temáticas y aspectos de las narraciones se remite a trabajos previos (EGEA; ARIAS, 2015).

La selección de este instrumento para el desarrollo de esta investigación se debe a la importancia que las narrativas poseen para la construcción de la historia, así como en la comprensión que sobre la historia transmite su lectura (WERTSCH, 1998; 2002; RÜSEN, 2005).

\section{Resultados: qué recuerdan nuestros estudiantes}

Teniendo en cuenta el conjunto de composiciones analizadas ( $\mathrm{n}=107)$, es interesante observar cómo el 43\% de las mismos $(n=46)$ se limitan a realizar una mera enumeración de datos históricos inconexos en los que, mediante nombre propios y hechos aislados, intentan realizar una descripción del periodo trabajado. Dicho tipo de narraciones, per se, carecen de cualquier categoría asociada a la relevancia histórica, asumiendo la historia como sucesión de hechos pero sin atribuir ningún tipo de relación causal a los mismos. Es decir, que para ellos parecen ser importantes pero no siempre pueden aportar las razones, por eso se diferencia entre las composiciones que son meramente enumeraciones de 
hechos o aquellas que intentan describir algún elemento añadido. La libre elección de unos hechos sobre otros, indica que están más presentes en su memoria. Como se puede imaginar, el alumnado no concibe todos los periodos por igual (Gráfico 1).

Gráfico 1 - Distribución de los textos elaborados diferenciando entre los narrativos y los meramente enumerativos.

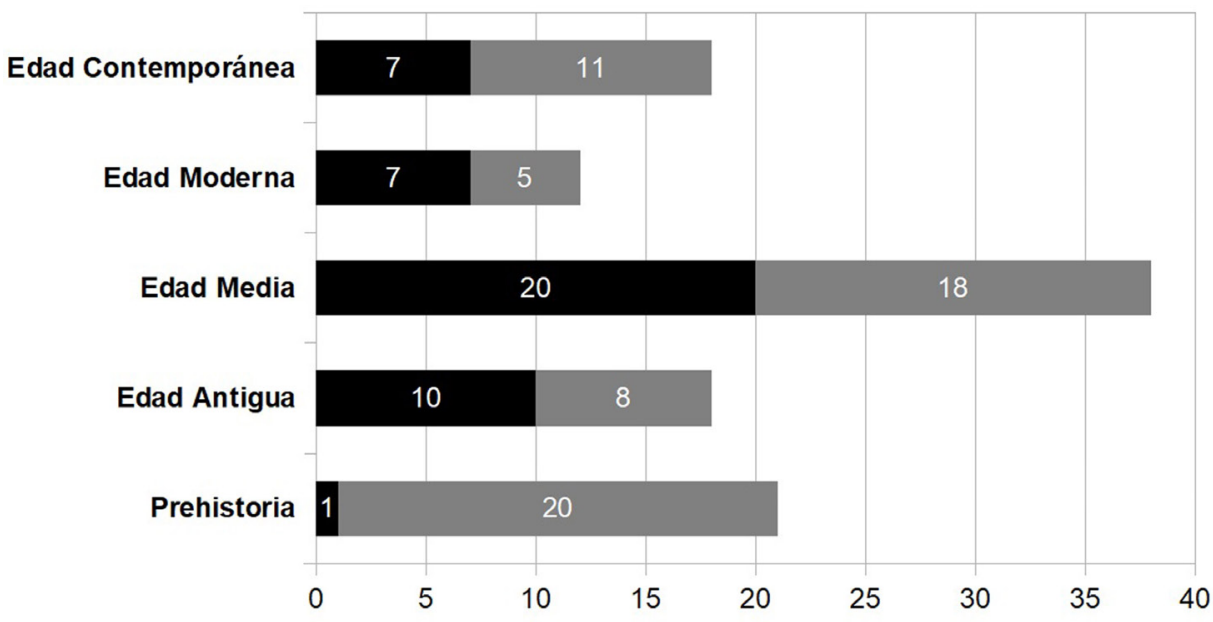

narraciones Descripciones v enumeraciones

Fuente: Elaboración propia.

Es interesante ver cómo a la hora de describir la Prehistoria, la ausencia de personajes de referencia o hechos concretos hace que el alumnado en general intente aproximarse a la misma de una manera más explicativa. En estos casos, la historia de la Prehistoria es narrada en términos de progreso desde el Paleolítico al Neolítico, estableciendo una comparación entre ambos periodos en cuestiones principalmente económicas, tecnológicas y sociales (modos de vida y alimentación).

[T106]3 Conocieron el suelo ¡Agricultura su consuelo!

[T014] El hombre cambió de nómada a recolector y la agricultura y ganadería apareció.

[T014] Con nuevas armas e ingeniería fue cambiando nuestra vida.

[T073] Creando herramientas el cultivo desarrollaron y los nómadas del mundo finalmente se asentaron.

Este sentido del discurso se ve modificado con la introducción del metal, al que otorgan un papel clave en la nueva configuración social, destacando el nuevo valor que este adquiere para la mejora del armamento y, como consecuencia, la aparición de la guerra.

3- A partir de aquí se incluye una selección de los textos [T] producidos por los estudiantes de $2 .{ }^{\circ}$ curso de Educación Primaria de la Universidad de Murcia. Este material ha sido recogido durante los años 2012 y 2015. 
[T048] Cobre, bronce, hierro, metales que forjan nuevas armas, nuevas guerras, pueblos que enfrentar.

La Edad Antigua, Media y Moderna se conciben principalmente como sucesión de datos inconexos. Escasas reflexiones sobre la trascendencia de los mismos se encuentran entre las líneas desarrolladas y siempre en términos del legado que esto supuso para la configuración del mundo actual.

En relación al mundo antiguo, estas alusiones se centran concretamente en influencia de la escritura, la democracia, los edificios de espectáculos fruto de la expansión romana $\mathrm{y}$ el derecho romano.

[T048] La escritura, grandes cambios, nueva etapa a comenzar.

[T045] Un sistema político se creó, llegó a ser tan famoso que a todo el mundo alumbró.

[T078] El imperio de Roma con su organización formó la cuna de nuestra civilización.

[T091] Dejándonos un gran legado, teatros, circos y anfiteatros.

[T084] No nos olvidamos de aquellos ciudadanos que nos aportaron el derecho romano.

La época medieval es entendida como un paréntesis en el discurrir de la historia. Escasa relevancia histórica atribuyen a los hechos que se incluyen en sus versos y se centran en dibujar un periodo caracterizado bien por la oscuridad y opresión (donde la Iglesia y la nobleza ejercen el control), bien como una época de conflicto (religioso y político) o, por el contrario, como una Edad Media mítica. Entre estas composiciones únicamente aparece una alusión al legado que las diversas culturas asentadas en la Península Ibérica aportaron en relación al devenir histórico de la misma.

[T009] Mezcla de culturas en terreno limitador, aportaciones diversas que nuestra historia ha condicionado.

Es curioso observar cómo incluso se llega a negar el contexto que rodea a determinados acontecimientos, atribuyendo al azar parte de su explicación.

[T067] Un nuevo camino hacia las Indias, por error encuentra nuevas tierras. Un continente que nace de una coincidencia.

En relación a la Edad Moderna, solo se cuenta con dos referencias a las posibles consecuencias derivadas de la política interior y exterior de algunos de los países europeos del occidente europeo.

[T104] Un imperio donde no se ponía el sol. Y durante dos siglos el mundo español habló.

[T080] Es una triste época de represión, en América esclavos, en España Inquisición.

Junto a esto, en una ocasión es mencionado el cambio de pensamiento de la época como motor de cambio, y la Revolución Francesa como momento de inflexión. 
[T071] Nace un nuevo pensamiento. El hombre es importante en el Renacimiento. Igualdad, libertad, fraternidad, esto son los ingredientes de la nueva sociedad.

[T093] La gente empezó a pensar, ya nada iba a ser igual. Tras la revolución francesa los derechos debían cambiar.

[T016] El pueblo francés buscaba libertad. Acabaron con los reyes para conseguir su igualdad (...) Fin del Antiguo Régimen, viva la Revolución.

Similar reflexión surge cuando un grupo de alumnos selecciona el final de la dictadura franquista como tema central de sus versos, enmarcado ya en la Edad Contemporánea. Es entonces cuando establecen la relación entre los movimientos que inundaron la España de los años 80, entre los que destaca por su estética la denominada Movida madrileña.

[T007] La Movida comenzó tras una dura represión, la gente salió a las calles para gritar “iLiberación!”.

Hasta aquí se han destacado únicamente aquellas reflexiones sobre relevancia histórica que, de manera expresa, señaló el alumnado. Pero existen otros aspectos que por su reiteración en las narraciones de los alumnos, se les debe dotar de cierta representatividad, aunque quizás no relevancia (Gráfico 2). Sin conocer los términos de su importancia, estos ocupan un lugar preeminente entre sus conocimientos sobre la historia universal.

Gráfico 2 - Episodios históricos más frecuentes a lo largo de las distintas etapas históricas. La línea discontinua marca el valor medio.

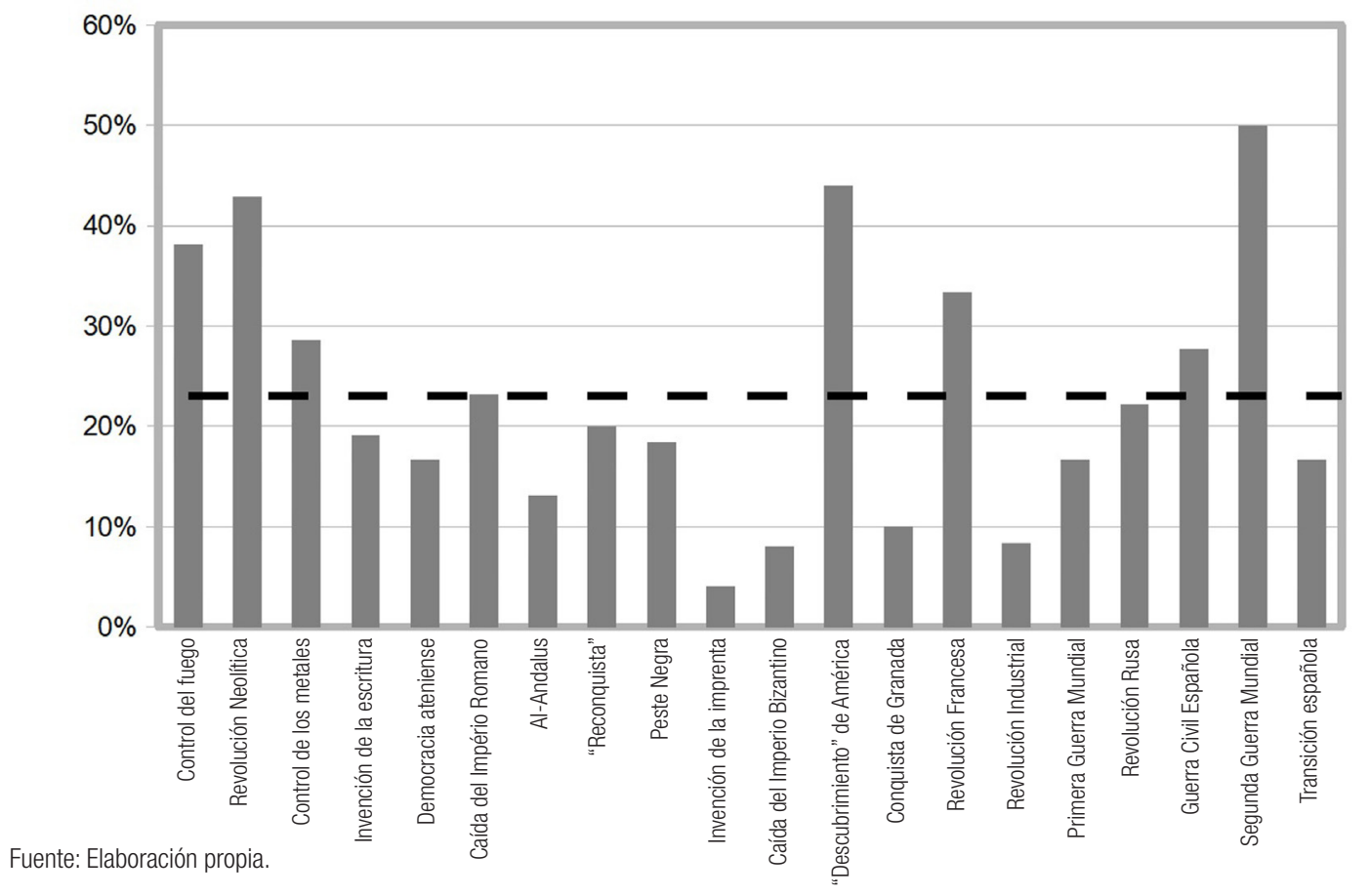


Para calcular esta representatividad se ha procedido a analizar cuántas narraciones hablaban de cada episodio en relación al período histórico correspondiente, comparando a posteriori las frecuencias de cada elemento respecto a su etapa. Sin embargo, para aquellos eventos que aparecen en ambas etapas, el cálculo se ha realizado respecto al número total de textos de ambas etapas. Por ejemplo, la Peste Negra ha aparecido en siete de los 38 textos sobre la Edad Media $(18,4 \%)$ y la Caída del Imperio Bizantino en cuatro de los cincuenta textos que sobre Edad Media y Edad Moderna existen (8\%).

Atendiendo a esto, por etapas históricas se constata que, para los alumnos, la Revolución neolítica, ya sea en relación a la aparición de la agricultura, la ganadería, la domesticación o la sedentarización, es uno de los elementos más recurrentes $(42,9 \%)$ de la Prehistoria.

De la Edad Antigua, la aparición de la escritura (19\%) o la democracia ateniense $(16,7 \%)$ son varias veces señalados pero bastante por debajo de la caída del Imperio romano $(23,2 \%)$. En este caso, cabe señalar que los hitos históricos que sirven de transición entre un periodo y otro suelen ser más frecuentes ya que aparecen en narraciones de sendas etapas.

Para la Edad Media, la denominada Reconquista de la Península Ibérica por parte de los cristianos (20\%) se muestra como uno de los hechos más referenciados, bastante por encima de la influencia de Al-Andalus (13,2\%). Pero, sin duda alguna, la llegada de los europeos a América es uno de los episodios más frecuentes (44\%) de toda la historia universal, en proporción sólo superado por el monopolio casi exclusivo que la Segunda Guerra Mundial posee durante la Edad Contemporánea (50\%). Alusiones a elementos de gran relevancia, aunque difícilmente detectable por el alumnado (como, por ejemplo, la invención de la imprenta) son residuales (4\%).

De la Edad Moderna, apenas hay información, ya que los conocimientos sobre el periodo son muy escasos. El episodio histórico estrella será la Revolución Francesa (33,3\%), de nuevo un episodio de transición. Cabe señalar una única mención a la Revolución industrial $(8 \%)$.

Finalmente de la Edad Contemporánea, como ya se adelantó, la Segunda Guerra Mundial aparece en repite en varias ocasiones, ya sea a través de sus protagonistas (Hitler, Stalin), de algunas de sus consecuencias (Holocausto, Bomba Atómica) o a través de los países beligerantes (Inglaterra, Alemania). Episodios como la Primera Guerra Mundial $(16,7 \%)$ o la Revolución rusa (22,2\%) completan la imagen que sobre el siglo XX posee el alumnado. Cabe añadir que sobre Historia de España casi los únicos elementos que serían relevantes para ellos desde 1492 serían la Guerra Civil española (27,8\%) y la Transición hacia la democracia (16,7\%). La España de los siglos XVI, XVII, XVIII y XIX parece no haber aportado nada al esquema histórico de los individuos investigados.

Por último, cabría reflexionar sobre otros textos que, debido al posicionamiento del alumnado, se puede entender que poseen una alta carga de significatividad histórica. Se está completamente de acuerdo con Levstik cuando afirmaba que desde el punto de vista del emisor (en su caso entrevistados, en el nuestro los redactores de las narrativas) "historical events took on significance when they 'formed us', 'changed us', or 'made us a nation”" (2000, p. 287). La posición que el alumnado toma ante la Historia nos hace ver 
también su forma de entender los mismos. Si bien los textos que se limitan a enumerar hechos no nos dan demasiadas claves para entender la perspectiva histórica que posee el alumnado participante en el estudio, sin embargo, sí que se han hallado una serie de composiciones, que se han venido a denominar como relatos $(n=28$, lo que constituye el 27\% del total) donde el alumnado se incluye en la narración como sujeto participante de la acción, utilizando para ello la primera persona (del singular o plural) mostrando un posicionamiento y actitud muy concreta respecto a determinados episodios de la historia, y un énfasis en temáticas concretas que destacan frente a otras.

En este sentido, al narrar la Prehistoria se incluyen como cazadores (cinco) o agricultores (tres), y, en una ocasión, como constructor de megalitos. Desde el punto de vista del sujeto, describen las herramientas, formas de vida y características del momento.

[T014] Con nuevas armas e ingeniería fue cambiando nuestra vida. Ya podíamos labrar, cosechar y al perro domesticar.

En la Edad Antigua, la identificación del sujeto oscila entre la presentación como romanos (tres) asumiendo el papel de gladiadores, guerreros o conquistadores, o como los pobladores de la Península Ibérica antes de la llegada de Roma (uno).

[T006] Arrasaremos Germania sin dejar un solo bárbaro.

[T045] Los romanos nos invadieron.

En relación a la Edad Media, se halla un mayor número de sujetos identificados con la Europa o España cristiana. Así, aparecen nobles (dos), caballeros (tres) y soldados (dos) cuyas hazañas principales se centran en su participación en las Cruzadas o en la que denominan como Reconquista. En general, cuando se posicionan siempre lo van a hacer en clave bélica.

[T036] En la Península estábamos de Reconquista, los hispanos recuperando su vida.

[T020] A un soldado nazarí maté durante la Reconquista. Y con Alfonso X el Sabio entramos en Sevilla.

[T009] Quisiera ser un noble, vivir en un castillo, poseer tierras, tener la ley en el bolsillo,tener en las catedrales mi lugar de exilio, defender a mi rey, luchar contra enemigos.

El viaje de Colón a América es de la misma manera el motivo perfecto para que dos grupos de alumnos se suban a sus barcos como exploradores.

[T005] Encontrarme entre mares, dejándome llevar, en 1492 América encontrar de la mano de Colón y con Santa María regresar a ver la Alhambra que a nuestras manos acababa de llegar.

[T069] En busca de las Indias, a tierra firme llegué, navegando navegando con América me encontré.

Por último, un único grupo se suma al movimiento reformista de la Iglesia, o se evade de la dictadura franquista en España. 
¿Qué es relevante históricamente? Pensamiento histórico a través de las narrativas de los estudiantes...

[T021] Me gustaría recorrer Europa con Erasmo. Ponerme en contra de la Iglesia. No seguir nunca más sus dictados y sentir como nace en mí el espíritu de protesta.

[T090] En conciertos de los Beatles me recreo mientras en mi país una dictadura nos censura.

\section{Discusión: qué es relevante para nuestros estudiantes}

A través de las narrativas de nuestros estudiantes, se han detectado ciertas referencias y reflexiones sobre algunos acontecimientos o episodios que, para ellos, han sido más determinantes que otros en el devenir de la historia de la humanidad o de nuestro país.

Para la Prehistoria, uno de los hechos más determinantes es el desarrollo de la industria lítica y la invención de la agricultura que son presentados como elementos de cambio que culminan con la sedentarización ([T014, T073]). La Edad de los Metales, por ejemplo, posee unas consecuencias claras, la invención de las armas y, por consiguiente, el desarrollo de las guerras ([T042, T048]). Para el mundo antiguo una de las escasas reflexiones sobre por qué un acontecimiento merece la pena ser recordado se encuentra en la aparición de la democracia en Atenas ([T045]) y su influencia actual en el mundo. La Revolución Francesa, por ejemplo, es otro de esos momentos determinantes para ellos. No solo por la cantidad de menciones sino porque en ocasiones ([T093]) se reflexiona sobre su papel en la historia como punto de inflexión a la hora de derechos sociales.

En muchas ocasiones, sin embargo, y a pesar de un alto número de menciones, la reflexión sobre el acontecimiento está completamente ausente. En el mundo antiguo, y en concreto el Antiguo Egipto no se detecta ni un solo elemento relacionado con la causalidad o la relevancia histórica, tratándose sin más de una suma de nombres propios. Otro caso muy claro es el que denominan como descubrimiento de América. Este se recuerda a la manera de un relato de aventuras, en ocasiones hasta ingenuo.

Atendiendo a los datos obtenidos, los estudiantes analizados, en principio, cuando establecen que algo es determinante en la historia lo hacen en los siguientes términos: 1) porque afectó a muchas personas; 2) porque duró mucho tiempo; 3) porque les afecta a ellos en la actualidad de un modo u otro o 4) porque el suceso supone un punto de inflexión en la línea temporal de la historia de la humanidad.

Pese a estas distinciones, la escasa aparición de elementos señalados (y la escasa relevancia asociada a los mismos), nos hace plantearnos la posibilidad de que realmente no sean capaces de comprender el alcance de muchos de los acontecimientos históricos que recuerdan. Las razones de esta circunstancia se comparten con Cercadillo, cuando constató que España, como la mayoría de los países europeos, con la excepción del currículum británico, "emphasize the pursuit of facts and details over questions of historical significance, evidence and interpretation” (2006, p. 8).

Se ha podido comprobar que, a grandes rasgos, el esquema temporal del futuro profesorado se cimenta sobre unos pilares o episodios históricos mínimos, la mayoría de ellos exclusivamente limitados a los episodios que marcan el cambio de etapa histórica. Se está ante un esqueleto muy reducido, ausente de músculos, tendones, prácticamente vacío.

Sorprende también la escasa aparición de determinados episodios que son claramente relevantes para la historia de habitantes del sur de Europa. Es el caso de la romanización, 
la expansión del Islam y la correspondiente influencia de Al-Andalus, por citar algunas ausencias muy significativas. Afirmaba Levstik que "silences in the history curriculum are reflections of silences in the larger culture” (2000, p. 297). El currículum español, así como el material curricular y libros de texto utilizados en las aulas, sí que incluye estos elementos, pero queda demostrado que no se les dedica el tiempo necesario o que están mayoritariamente ausentes de las narrativas maestras, tan influyentes a la hora de transmitir y concebir la historia (LÓPEZ RODRÍGUEZ, 2015).

Y es que, no se puede obviar que, a pesar de todos nuestros esfuerzos como docentes, otros factores externos como los medios de comunicación, el contexto social, el grupo, la familia, la propia relación entre discentes y docentes, etc., determinan en numerosas ocasiones lo que se recuerda de historia y aquello que se piensa que es más importante (EPSTEIN, 1997; WINEBURG, MOSBORG; PORAT, 2001). Cabe también plantear como causa de algunas omisiones o faltas, la dicotomía que Wertsch (1998; 2002) planteó a la hora de equiparar las narrativas históricas como ejemplo de herramientas culturales que nos sirven para realizar acciones mediadas. El hecho de que nuestros alumnos hayan dominado (mastery) unos contenidos, unas narrativas, así lo hicieron para superar sus cursos, no implica que las hayan asimilado (appropriation) como propias.

\section{Conclusiones e implicaciones educativas y de investigación}

Como se ha podido comprobar para los estudiantes analizados hay ciertos acontecimientos que son más relevantes históricamente que otros. Sin embargo, se detecta una escasez preocupante en cuanto a las razones para elegir unos hechos sobre otros. Es decir, que memorizan pero no reflexionan, no piensan históricamente. Decía Seixas que era crucial no solo enseñar historia sino someter a crítica las estrategias que se estaban empleando a la hora de transmitir la relevancia histórica a los estudiantes (SEIXAS, 1994) ya que era uno de esos "epistemological minefields through which there is no one simple, well-beaten path” (SEIXAS, 1996, p. 778), pero que, a partir de esta dificultad, la labor de los investigadores en didáctica de la Historia debía ser aclarar el camino para que profesores y estudiantes no cayesen heridos durante el proceso. Observando las carencias que sobre estos aspectos poseen los futuros docentes se hace urgente, ampliando el símil del profesor canadiense, que todos los artificieros se pongan manos a la obra. Para ello son necesarios estudios de profundidad sobre la temática.

La realidad es que, como afirmaban Seixas y Morton (2013), la significance en Historia, varía a lo largo del tiempo y de grupo a grupo y, aún más, dependerá de la edad y la formación histórica recibida (SEIXAS, 1994). No se puede llegar en esta investigación a reflexionar sobre qué grado de comprensión o explicación de la significatividad en la historia poseen los individuos analizados, como han realizado otros estudios, pues las narrativas entremezclan sin distinción lo históricamente determinante, con lo curioso, lo raro, lo anecdótico. Sin embargo, sí se pueden esbozar las reflexiones y consideraciones que sobre esta categoría y en relación al devenir de la historia de la humanidad o de nuestro país poseen nuestros estudiantes en la actualidad. Seixas (1997) distinguió entre posturas objetivistas básicas, subjetivistas básicas, objetivistas avanzadas, subjetivistas 
avanzados y la narrativista. Por su parte, Cercadillo (2000), diferenció entre explicaciones contemporáneas, causales, de patrón, simbólicas, reveladoras o presentistas. Ulteriores pruebas, narrativamente más extensas, podrían precisar algo más en cuanto a estos distintos niveles de comprensión de la relevancia histórica. En nuestro caso, con la brevedad de los textos analizados únicamente se puede distinguir que un buen número de alumnos consideraron que merecía la pena recordar algunos acontecimientos y no otros, hitos importantes en su opinión y, por tanto, determinantes históricamente, dando incluso las razones de esta elección. Se puede concluir que, gracias a esta investigación, se han identificado qué acontecimientos históricos creen los futuros docentes de Educación Primaria que tienen más relevancia histórica y que, en un futuro, gracias a conocer la profundidad con la que nuestros estudiantes se enfrentan a la comprensión de algunos de estos periodos se podrán establecer las pautas a seguir para mejorar la acción docente.

Las ventajas de llevar al aula estrategias y actividades que trabajen lo históricamente determinante y fomenten su comprensión, serán a la larga muy beneficiosas. Hunt (2000) defiende que si se enfatiza este aspecto de la enseñanza de la historia se producirá un cambio sustancial en la actitud del alumnado al comprender el valor de su estudio. Ya hay algunas experiencias de aula muy interesantes sobre el tema (HAMMOND, 2001; COUNSELL, 2002; CONWAY, 2006; WRENN, 2011) e incluso algunas de ellas constataron que, a raíz de su implementación, se produjo una mejora en la motivación, las preguntas de los alumnos fueron más reflexivas y más significativas, cambió la percepción sobre algunos temas aparentemente menos interesantes y los lazos entre lo local, lo nacional y lo internacional se estrecharon (PHILLIPS, 2002).

Manuales repletos de datos ya seleccionados y preparados, procesos de evaluación básicamente centrados en lo conceptual, condiciones laborales mejorables para el profesorado (ratio, diversidad, aulas, horario) son algunas de las razones que bloquean la llegada de una nueva manera de enseñar y aprender historia en las aulas españolas. Todo ello determinado, en nuestra opinión, por lo inabarcable del currículum que hace imposible un enfoque adecuado por parte del docente. La situación en el caso de España se complica más aún con la nueva reforma educativa (LÓPEZ-FACAL, 2014).

Todos estos factores dificultan plantear, sin duda alguna, clases de Historia donde el porqué de cada tema impartido esté meridianamente claro tanto para el discente como para el docente. Aún así, se hace necesario focalizar cada uno de los periodos en base a ciertos interrogantes que lleven, a la larga, a comprender la historia de una manera más global y crítica, además de que, clarificando lo históricamente relevante, puede aumentar exponencialmente la motivación y el gusto hacia la disciplina entre nuestro alumnado. 


\section{Referencias}

BARTON, Keith; LEVSTIK, Linda. 'It wasn't a good part of history': national identity and students' explanations of historical significance. Teachers College Record, New York, v. 99, n. 3, p. 478-513, 1998.

CERCADILLO, Liz. 'Maybe they haven't decided yet what is right:' English and Spanish perspectives on teaching historical significance. Teaching History, London, n. 125, p. 6-10, 2006.

CERCADILLO, Liz. Significance in history: students' ideas in England and Spain (Phd). London: Institute of Education/University of London, 2000. Disponible en: <http://eprints.ioe.ac.uk/6632>. Acceso en: 30 abr. 2015.

CONWAY, Robin. What they think they know: the impact of pupils' preconceptions on their understanding of historical significance. Teaching History, London, n. 125, p. 10-15, 2006.

COUNSELL, Christine. Looking through a Josephine-Butlershaped window: focusing pupils' thinking on historical significance. Teaching History, London, n. 114, p. 30-36, 2002.

DOMÍNGUEZ, Jesús. Pensamiento histórico y evaluación de competencias. Barcelona: Graó, 2015.

EGEA, Alejandro; ARIAS, Laura. Russell Crowe el gladiador, Colón el descubridor y otros protagonistas de la historia: buceando en las narrativas históricas de jóvenes universitarios españoles. Clio, Zaragoza, n. 41, 2015. Disponible en: <http://clio.rediris.es>. Acceso en: 30 abr. 2015.

EPSTEIN, Terrie. Sociocultural approaches to young people's historical understanding. Social Education, Silver Spring, v. 61, n. 1, p. 28-31, 1997.

GÓMEZ, Cosme; ORTUÑO, Jorge; MOLINA, Sebastián. Aprender a pensar históricamente: retos para la historia en el siglo XXI. Tempo e Argumento, Florianópolis, v. 6, n. 11, p. 5-27, 2014. Disponible en: <http:// doi.org/10.5965/2175180306112014005>. Acesso en: 28 nov. 2015.

HAMMOND, Kate. From horror to history: teaching pupils to reflect on significance. Teaching History, London, n. 104, p. 15-24, 2001.

HENRÍQUEZ, Rodrigo; PAGÉS, Joan. La investigación en didáctica de la Historia. Educación XX1, Madrid, v. 1, n. 7, p. 63-83, 2004. Disponible en: <http://doi.org/10.5944/educxx1.7.1.330>. Acesso en: 2 nov. 2015.

HUNT, Martin. Teaching historical significance. In: ARTHUR, James; PHILLIPS, Robert (Ed.). Issues in history teaching. London; New York: Routledge, 2000. p. 39-53.

LÉVESQUE, Stephan. Teaching second-order concepts in Canadian history: the importance of "historical significance". Canadian Social Studies, Edmonton, v. 39, n. 2, 2005. Disponible en: <http://www.educ. ualberta.ca/css/Css_39_2/ARLevesque_second-order_concepts.htm>. Acceso en: 25 sept. 2015.

LÉVESQUE, Stephan. Thinking historically educating students for the twenty-first century. Toronto: University of Toronto Press, 2008. 
LEVSTIK, Linda. Articulating the silences: teachers' and Adolescents' conceptions of historical significance. In: STEARNS, Peter; SEIXAS, Peter; WINEBURG, Sam (Ed.). Knowing, teaching and learning history: national and international perspectives. London; New York: New York University Press, 2000. p. 284-305.

LEVSTIK, Linda. Learning history. In: MAYER, Richard; ALEXANDER, Patricia (Ed.). Handbook of research on learning and instruction. New York: Routledge. 2011. p. 108-26.

LOMAS, Tim. Teaching and assessing historical understanding. London: Historical Association, 1990.

LÓPEZ-FACAL, Ramón. La LOMCE y la competencia histórica. Ayer, Madrid, v. 94, n. 2, p. 273-385, 2014.

LÓPEZ RODRÍGUEZ, César. Repensando las narrativas nacionales: un análisis del origen, transmisión e influencia en el aprendizaje histórico. Panta Rei, Murcia, p. 77-92, 2015.

PARTINGTON, Geoffrey. The idea of an historical education. Windsor: NFER, 1980.

PHILLIPS, Robert. Historical significance- the forgotten "Key Element"? Teaching History, London, n. 106, p. 14-19, 2002.

RÜSEN, Jörn. History: narration, interpretation, orientation. New York: Berghahn Books, 2005.

SEIXAS, Peter. Conceptualizing growth in historical understanding. In: OLSON, David; TORRANCE, Nancy (Ed.). The handbook of education and human development: new models of learning, teaching and schooling. Malden: Blackwell, 1996. p. 765-783.

SEIXAS, Peter. Mapping the terrain of historical significance. Social Education, Washington, v. 61, n. 1, p. 22-27, 1997.

SEIXAS, Peter. Students' understanding of historical significance. Theory \& Research in Social Education, Silver Spring, v. 22, n. 3, p. 281-304, 1994.

SEIXAS, Peter.; MORTON, Tim. The big six: historical thinking concepts. Toronto: Nelson Education, 2013.

SEIXAS, Peter; PECK, Carla. Teaching historical thinking. In: SEARS, Alab; WRIGHT, lan (Ed.). Challenges and prospects for canadian social studies. Vancouver: Pacific Educational Press, 2004. p. 109-117.

STRAUSS, Anselm; CORBIN, Juliet. Basics of qualitative research: techniques and procedures for developing grounded theory. Thousand Oaks: Sage, 1998.

VANSLEDRIGHT, Bruce. Assessing historical thinking and understanding: innovative designs for new standards. New York: Routledge, 2014.

VANSLEDRIGHT, Bruce. The challenge of rethinking history education: on practices, theories, and policy. New York: Routledge, 2011.

WERTSCH, James. Mind as action. New York: Oxford University Press, 1998. 
WERTSCH, James. Voices of collective remembering. Cambridge; New York: Cambridge University Press, 2002.

WINEBURG Sam. Historical thinking and other unnatural acts: charting the future of teaching the past. Philadelphia: Temple University Press, 2001.

WINEBURG Sam. Unnatural and essential: the nature of historical thinking. Teaching History, London, n. 129, p. 6-11, 2007.

WINEBURG, Sam; MOSBORG, Susan; PORAT, Dan. What can Forrest Gump tell us about students' historical understanding? Social Education, Silver Spring, v. 1, n. 65, p. 55-58, 2001.

WRENN, Andrew. Significance. In: DAVIES, lan (Ed.). Debates in history teaching. Abingdon, Oxon; New York: Routledge, 2011. p. 148-158.

Recibido en: 31.08.2016

Aprobado en: 23.11.2016

Laura Arias Ferrer es doctora en Historia por la Universidad de Alicante y licenciada en Historia por la Universidad de Murcia. Profesora contratada doctora de Didáctica de las Ciencias sociales de la Facultad de Educación de la Universidad de Murcia, España. Miembro del grupo de investigación DICSO y secretaria del CEPOAT.

Alejandro Egea Vivancos es doctor en Historia y licenciado en Geografía e Historia por la Universidad de Murcia. Profesor contratado doctor de Didáctica de las Ciencias sociales de la Facultad de Educación de la Universidad de Murcia, España. Miembro del grupo de investigación DICSO y subdirector del CEPOAT. 